\title{
A Service-Oriented Framework for Parallel Medical Image Reconstruction ${ }^{\star}$
}

\author{
S. Benkner, A. Dimitrov, G. Engelbrecht, R. Schmidt, and N. Terziev \\ Institute for Software Science, University of Vienna, \\ Liechtensteinstrasse 22, A-1090 Vienna, Austria \\ \{sigi, dimitrov, gerry, rainer, terziev\}@par.univie.ac .at
}

\begin{abstract}
This paper presents an overview of the eMERGE system, a service-oriented system for medical image reconstruction on parallel computers. Within the eMERGE system, parallel image reconstruction codes running on SMP clusters or other parallel machines may be exposed as Jini services, as Web Services, or as OGSA services and accessed over the Internet by means of a browser-based GUI. The system provides support for dynamic service management, service selection and monitoring. We describe the parallelization of a fully $3 \mathrm{D}$ iterative image reconstruction code using a combination of MPI and OpenMP, discuss the major design and implementation issues of the various service types supported by our system, and present experimental results comparing the performance of RMI-based to SOAP-based image data transfers.
\end{abstract}

\section{Introduction}

Novel algorithms enable very accurate 3D reconstruction of medical images from 2D scanner data by considering principal 3D effects of data acquisition. However, the high computational requirements restrict the deployment of these methods to dedicated research centers. In order to make advanced image reconstruction services available to hospitals that have no in-house high performance computing facilities, we are developing the eMERGE system, a service-oriented framework that supports near real-time 3D image reconstruction by providing transparent access to remote parallel computers over the Internet. Our current prototype consists of a browser-based GUI, a parallel fully 3D image reconstruction code written in $\mathrm{C} / \mathrm{MPI} / \mathrm{OpenMP}$, and a framework for dynamic service management, service selection and monitoring. Parallel image reconstruction codes provided on PC clusters or other parallel machines may be exposed in our system as Jini services, as Web Services, or as OGSA-compliant Grid services.

This article is organized as follows: Section 2 provides an overview of the image reconstruction algorithm and its parallelization. Sections 3 and 4 describe the main components of the eMERGE system and the different service variants,

\footnotetext{
* This research was partially supported by the Austrian Science Fund as part of the AURORA project under contract SFB F1102 and by the European Commission as part of the IST Project GEMSS under contract IST-2001-37153.
} 
respectively. Performance results of image transfers using different protocols are presented in Section 5, followed by conclusions and future plans in Section 6 .

\section{Parallel Fully 3D Image Reconstruction}

In emission tomography (e.g. SPECT), the spatial distribution of a radioactive tracer is reconstructed from projection data. In fully $3 \mathrm{D}$ image reconstruction a solution for the whole image volume $X$ is found simultaneously by using the whole set of projection values $Y$. The imaging process is characterized by the following equation:

$$
Y=A X+E
$$

The image volume $X$ is represented by means of a vector of size $J$ which stores the mean activity of each voxel. Vector $Y$ of size $I$ represents the whole set of projection values, counting the photons detected for each projection value during the whole acquisition. Element $a_{i j}$ of the system matrix $A$ represents the probability that a photon emitted in voxel $j$ is detected at detector position $i$. The system matrix allows a flexible modeling of scanner geometry, detector efficiency, scatter and attenuation. In typical clinical applications $J$, the number of unknowns is $128^{3}$ and $I$, the number of equations is $128^{2} \times 120$ assuming $3^{\circ}$ rotational increment over a range of $360^{\circ}$.

The ill-posedness of $A$ and the error term $E$, in general, allow no exact solution of Eqn. 1. In our system we utilize an improved variant of the wellknown ML-EM algorithm for emission tomography [12], which is an iterative method for finding a feasible solution of Eqn. 1. The ML-EM algorithm takes into account the Poisson statistics of photon emission and detection. During one iteration step, the voxel $x_{j}^{(n)}$ of the $n$-th iteration is updated by

$$
x_{j}^{(n+1)}=x_{j}^{(n)} \sum_{i} a_{i j} \frac{y_{i}}{\sum_{k} a_{i k} x_{k}^{(n)}} .
$$

A more detailed description of our reconstruction algorithm, which implicitly corrects for scatter and attenuation, can be found in [2].

Experiments with a sequential implementation of the image reconstruction algorithm in $\mathrm{C}$, have shown that the time required for the reconstruction of a $128^{3}$ image volume on a single processor workstation can be in the range of several hours to a few days, depending on the configuration of the algorithm and the hardware.

\subsection{Parallelization for SMP Clusters}

In order to allow on-site clinical analysis the reconstruction code has been parallelized. Since our main target platforms are SMP clusters, we adopted a hybrid parallelization strategy relying on a combination of MPI [7] and OpenMP [11]. Distributed-memory parallelization, based on the single-program multiple-data (SPMD) paradigm, is achieved by parameterizing the code in such a way that it 
can be executed by multiple MPI processes, each computing its own part of the image volume. This was implemented by strip-mining the outer loop representing the computations defined by Eqn.2 across multiple processes. By utilizing appropriate OpenMP work sharing constructs, each MPI process employs multiple OpenMP threads. At the end of a full iteration cycle, the voxels updated by individual MPI processes are transferred to all other processes in a collective communication phase.

Since most of the elements of the system matrix are zero we utilize the compressed row storage format (CRS) in our implementation. Moreover the symmetries of the system matrix are exploited to further reduce the storage requirements. In order to minimize communication overheads, the system matrix and some other data structures are allocated on each node.

The parallelized source program is compiled with an OpenMP C compiler and linked with the MPI library. The resulting program is executed on an SMP cluster as an MPI program by a set of parallel processes, each usually running on a separate node of the cluster within its own local address space. Due to the use of OpenMP, each MPI process employs multiple threads (usually one thread per processor) which execute concurrently in the shared address space of a node.

The combination of MPI and OpenMP offers greater flexibility with respect to memory management than an approach based on MPI only. Moreover, due to the shared memory available within nodes, the number of communications can be reduced while message sizes can be increased. In a pure MPI implementation of our algorithm, not only more messages but also much more memory is required. On the other hand, by using OpenMP, certain synchronization overheads are introduced whenever multiple threads update data structures allocated in the shared memory of a node. Experimental results comparing hybrid-parallel versions of the reconstruction algorithm to a pure message passing version are presented next.

\section{$2.2 \quad$ Performance Experiments}

We present performance experiments with different versions of the parallel image reconstruction code on a Beowulf-type PC cluster consisting of 6 nodes connected by Fast Ethernet. Each node is equipped with 4 Pentium III Xeon processors $(700 \mathrm{MHz})$ and $4 \mathrm{~GB}$ of memory. For the compilation of the MPI/OpenMP source program the $\mathrm{C}$ compiler from Portland Group Inc. was used together with the mpich library.

In Figure 1 the speed-ups (with respect to the sequential code executed on a single processor of the cluster) of three different code variants are compared for an image of size $128 \times 128 \times 128$ using data from a clinical standard phantom. A pure MPI version is compared to hybrid-parallel versions which employed 2 and 4 threads within each MPI process, respectively. The hybrid-parallel versions have been executed in such a way that each thread is always running on its own processor. On up to two nodes (8 processors), all three versions yield approximately the same speed-ups. On more than two-nodes, the pure MPI-version 


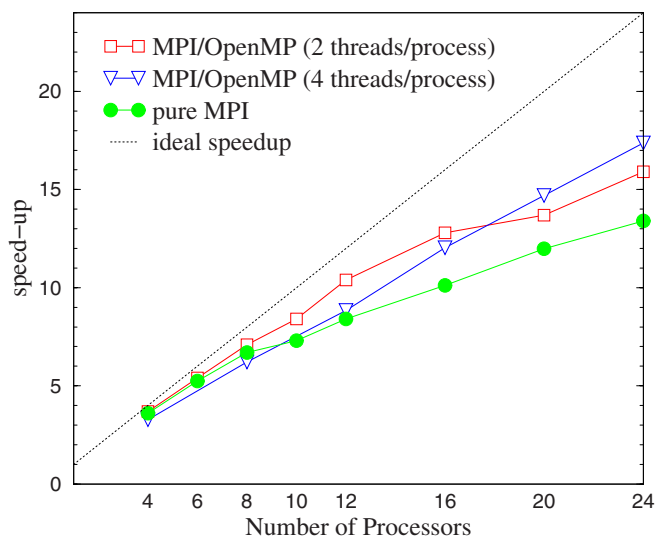

Fig. 1. Experimental comparison of a pure MPI version to hybrid-parallel MPI/OpenMP versions with 2 and 4 threads per MPI process, respectively.

is outperformed by the MPI/OpenMP versions. One reason for the worse scaling of the pure MPI version is the larger communication overhead (more and smaller messages), since communication is performed also within nodes. Moreover, the MPI version requires more memory because replicated data structures are allocated within each process, while in the MPI/OpenMP version all threads have access to the data allocated within their parent processes. As can be observed from Figure 1, the performance of the MPI/OpenMP version where only 2 threads were generated within each MPI process decreases when more than one MPI process is run on a node, i.e. if more than 12 processors are used. If all 24 processors of the cluster are utilized, a significant performance difference between the pure MPI version and the MPI/OpenMP versions can be seen.

Our reconstruction algorithm has been implemented in such a way that it is portable across distributed-memory architectures, shared-memory architectures and clusters. A performance evaluation on other parallel computing platforms, including a heterogeneous SMP cluster (2-processor nodes and 4-processor nodes), an SGI Origin, and an NEC SX-5 multi-node vector supercomputer is currently under way. In order to efficiently utilize the computing power offered by vector supercomputers such as the SX-5, modifications of our implementation will be required for exploiting all three levels of parallelism (i.e. distributedmemory, shared-memory, and vector parallelism).

\section{The eMERGE System}

Within the eMERGE system, parallel image reconstruction codes running on PC clusters may be exposed as Jini services, as Web Services, or as OGSA-compliant Grid services. The eMERGE system provides basic support for automatic service selection, dynamic service management, and service monitoring. Services may be accessed via a browser-based graphical user interface either in direct mode, in 



Fig. 2. Main components of the eMERGE system.

which case a predefined reconstruction service is accessed, or in managed mode, where a service manager automatically selects from the set of available parallel machines the one on which the reconstruction job is expected to be completed within the shortest period of time. The main components of the eMERGE system are shown in Figure 2.

In a typical use-case scenario, projection data from a CT scanner are loaded from the client's local file system and displayed in the GUI. The user selects the slices to be reconstructed and various other reconstruction parameters. When the user decides to start the reconstruction process, the raw image and an XML file, which contains meta data about the image and the reconstruction process, are transferred over the Internet to a remote parallel machine. On the target machine, an intermediate Java program handles the transfer of image data, and initiates the execution of the native image reconstruction code. By monitoring the reconstruction process, the user is provided with an estimated service completion time and corresponding progress information. After completion of the reconstruction process, the reconstructed 3D image is transferred back to the client and displayed in the GUI.

\subsection{Reconstruction Services and Server-Side Components}

The native image reconstruction code, written in $\mathrm{C} / \mathrm{MPI} / \mathrm{OpenMP}$, may be installed on PC clusters and other parallel computers and exposed as a service through an intermediate server-side Java program, called service executor, which handles the data transfer between clients and services, and the execution of the reconstruction job on the parallel target machine. The service executor implements the interface ReconstructionService, which contains all methods a client may invoke on an image reconstruction service. The service executor relies on a service monitor for monitoring the state of a running service, and a machine monitor for obtaining an estimate of the available computational power of the target machine at a certain point in time. For this purpose the machine monitor needs access to the local batch system. Upon start-up, the machine monitor is configured with an XML machine descriptor, which currently contains the number of available processors as well as their estimated performance.

In order to support the concurrent execution of multiple reconstruction jobs on the same target machine, the service executor generates a unique id for each 
client, which is passed as an argument to all methods provided by the reconstruction service. As a consequence, a single instance of the service executor can be accessed by multiple clients at the same time.

\subsection{Service Manager and Service Registry}

Reconstruction services may be accessed in direct mode or in managed mode. In direct mode, the client has to know the location of the service. In managed mode, the client contacts a service manager, which dynamically selects a reconstruction service to be accessed by the client. Whenever a reconstruction service is added to the system, the service registers itself in the service registry. Upon an incoming client request, the service manager selects from the set of registered services the one that is expected to complete the reconstruction job within the shortest period of time. For this purpose, the service manager maintains state information of all registered service machines and chooses the machine that provides the maximum free computational capacity (currently measured as the accumulated performance of free processors) at the time a service is requested. Whenever the number of available free processors on a registered service machine changes, the service manager receives a corresponding notification from the machine monitor. After having selected a service, the service manager forwards an appropriate service proxy to the client which then accesses the chosen service directly.

\subsection{User Interface and Client-Side Components}

The image reconstruction service system may be accessed via a graphical user interface that can be provided to end users as a Java applet via a browser. Since the GUI needs to access the client's local file system for loading and saving medical images, we utilize a signed applet which presents a X.509 certificate upon download to the user for granting the required permissions.

The GUI utilizes a flexible service connector component which is capable of loading or generating different service proxies in order to access reconstruction services via RMI or Web Services (JAX-RPC, SOAP) protocols.

\section{Service Technologies}

Initially, the eMERGE system has been developed based on Jini. Support for Web Services and OGSA has been added recently and is a major objective of ongoing work.

\subsection{Jini}

Within the Jini-based implementation of the eMERGE system, reconstruction codes installed on parallel computers are exposed as services accessible via the Java RMI protocol. Using the basic mechanisms of Jini (discovery, join and lookup), a dynamic federation of multiple image reconstruction services on different parallel hosts may be established. In such a federation of services, at least 
one service registry (realized as a Jini lookup service) and one service manager (realized as a Jini service) must be instantiated. Whenever a reconstruction service is added to the system, the service registers itself with the service registry. The service manager obtains service proxies of all available reconstruction services from the service registry.

Upon startup of the client GUI, the service connector component discovers the service registry and obtains a proxy object of the service manager. At the time a client issues a request for an image reconstruction service, the service manager selects a suitable image reconstruction service (see below) and returns the proxy of the corresponding service executor to the client. The client's service connector then accesses the image reconstruction service directly. The raw image data and the XML-based meta-data are transferred over RMI to the target machine by calling appropriate methods of the service executor. Thereupon, the service executor launches the parallel reconstruction process on the target machine, usually utilizing all available free processors.

At the moment our service manager uses a fairly simple strategy for selecting a reconstruction service: the machine with the maximum available computing resources, currently measured as the number of free processors times the estimated processor performance in FLOPs, is selected. In order to provide up-todate information about the actual free computational capacity of a machine, the monitoring component notifies the service manager whenever the number of free processors changes.

In order to support realistic settings, where server-side machines as well as clients are behind firewalls, we utilize HTTP tunneling of all RMI calls.

\subsection{Web Services}

Web Services are rapidly becoming the standard way of performing Enterprise Application Integration (EAI) within as well as across organizations. Web Services are based on XML vocabularies standardized by the W3C (SOAP, WSDL, UDDI, etc.) and provide an architecture for exposing applications as services that are platform and programming language independent. Web services are identified by an URI, expose their public interfaces and protocol bindings through a Web Services Description Language (WSDL) document, and are usually accessed via SOAP over HTTP, even across firewalls.

Our current Web Services implementation of image reconstruction services supports access in direct mode only. For the development we mainly used the Java Web Service Developer Pack (JWSDP 1.0 [8]) which provides a framework for developing and hosting web services based on servlets and Tomcat. Moreover, we have utilized other Web Services frameworks, mainly for the purpose of performance comparisons, including Glue [5] and Apache Axis [1].

The starting point for realizing image reconstruction services as Web Services is the Java interface ReconstructionService, which specifies all methods clients may invoke on a reconstruction service. In order to support access to reconstruction services based on Web Services technologies, a WSDL document that describes the public interfaces and protocol bindings is required. This WSDL 
document has been generated automatically from the Java interface provided for reconstruction services by using the xrpcc tool of the JWSDP. Besides generating a WSDL document from a Java interface, the xrpcc tool also generates stubs and ties for marshalling of arguments on the client-side and server-side, respectively.

On the client-side, we provide an additional service connector for accessing reconstruction services over JAX-RPC (which is SUN's implementation of SOAP RPC). The service connector loads the stub class of the reconstruction service over HTTP (by means of an URLClassLoader). Upon method invocation, the stub creates a SOAP request message that is conveyed via HTTP to a servlet on the server side. The servlet parses the request message, converts arguments from XML to Java and invokes the corresponding method of the service executor. The method's result object is transformed into an appropriate SOAP response message by the servlet, transferred back to the client, and transformed by the stub into a Java object.

Compared to a pure Java/RMI communication protocol, SOAP calls imply an additional overhead for marshalling arguments and for parsing SOAP messages both on the client side and the server side (see Section 5).

In addition to the stub-based service connector, we have implemented a connector that makes use of the Web Services Invocation Framework (WSIF 2.0 [13]) in order to access reconstruction services independently of the actual implementation and deployment as described in the next section.

\subsection{Open Grid Service Architecture}

We have implemented a variant of the medical image reconstruction service according to the Open Grid Service Architecture (OGSA) based on the Open Grid Service Infrastructure (OGSI) Application Framework (OGSI TP-5 [10]).

An OGSA service is a Web Service that provides standard mechanisms for creating, naming and discovering services [9]. These mechanisms are realized by an OGSI container which provides the runtime environment for Grid services. The OGSI container itself makes use of the Tomcat servlet engine and of Apache Axis. The OGSA specification requires that a compliant Grid service implements at least the interface (PortType) GridService, which is the base interface definition in OGSA.

As in the Jini and Web Services implementation, the OGSA implementation of the medical image reconstruction service is based on the Java interface ReconstructionService from which a WSDL document is generated. In order to provide an OGSA compliant implementation, our service executor component extends the class PersistentServiceSkeleton provided by the OGSI application framework implementing the interface (PortType) GridService. Moreover, a deployment descriptor has to be provided, specifying the URI of the service and other properties. Upon deployment, the service is automatically registered in the registry of the OGSI container.

At the current stage of implementation, the OGSA-compliant version of the service can be used in direct mode only. That is, the client application must 
know the service endpoint, which is an URI that designates the WSDL of the reconstruction service (also referred to as Grid Service Handle (GSH) in OGSA terminology). In order to access the OGSA service from our GUI, we have implemented a service connector based on the Web Service Invocation Framework (WSIF 2.0) [13]. Using WSIF, the service connector constructs a SOAP request based on the grid service reference (GSR) of the reconstruction service (which is the WSDL document of the service decorated by the container with runtime specific information) without the need of any OGSA specific classes or stubs. Another major advantage of WSIF is that besides SOAP other bindings and transport protocols may be supported (eg. EJB using RMI/IIOP).

\section{$5 \quad$ Experimental Results}

In this section we present performance results of image data transfer based on Java RMI in comparison to various implementations of SOAP (XML-RPC).
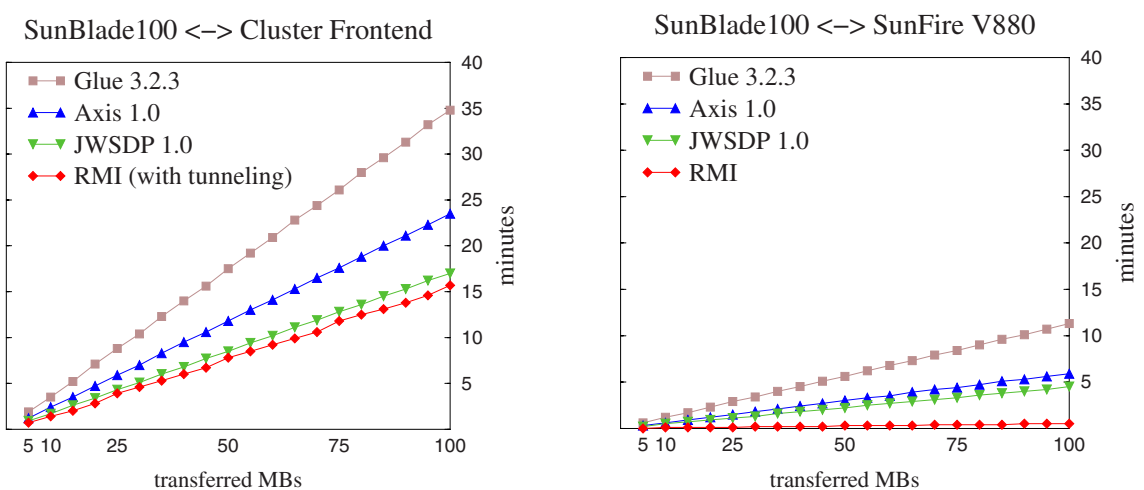

Fig. 3. Performance of data transfers: RMI vs. different SOAP implementations.

Although in our current reconstruction service the size of medical images is only approximately $5 \mathrm{MBs}$, we have measured transfers of up to $100 \mathrm{MBs}$ on a Fast Ethernet back and forth (ping-pong) between a client and a reconstruction service executor. The client application was executed on a Sun Blade 100 (sparcv9, 502MHz, 640MB RAM). The service executor was hosted on the front-end PC (dual Pentium II, 400MHz, 1GB total RAM) of our 64 processor SMP cluster (see left hand side of Fig. 3) and on a Sun Fire V880 (four sparcv9, 750MHz, 8GB RAM), respectively. Apparently for both settings, RMI data transfers are the fastest, followed by JAX-RPC with JWSDP [8], SOAP XML-RPC with Axis [1], and finally Glue's implementation [5] of SOAP-RPC. Note that in the figure on the left hand side we utilized HTTP tunneling of RMI calls in order to get access to the cluster frontend across the firewall. The overhead of SOAP based data transfers compared to RMI is mainly caused by 
the generation and parsing of SOAP messages and to a much lesser extent by the conversion of image data represented as a Java byte array to/from XML.

\section{Conclusions}

In this paper we gave an overview of a service-oriented system that provides access to parallel image reconstruction codes on different parallel computers via the Internet based on RMI and SOAP. We are currently extending our system to provide support for automatic service selection for the Web Services and OGSAbased service implementations. Moreover our Jini-based service manager is being extended in order to allow transparent access to a local Jini federation through Web Services or OGSA protocols similar to the ICENI system [6].

Major directions of future work will include the extension of the service manager with more sophisticated methods for automatic service selection based on user-specified criteria, support for authorization and authentication, and for secure data transfers. Our services will be integrated into a larger Grid-enabled Medical Simulation System which is currently being developed within the EU project GEMSS. Within GEMSS, negotiable QoS support for medical services and security of patient data under the constraints of EU legislation will be of major importance.

\section{References}

1. Apache Axis. http://ws.apache.org/axis

2. W. Backfrieder, S. Benkner, G. Engelbrecht. Web-based parallel ML-EM reconstruction for SPECT on SMP clusters, Proc. METMBS'01, CSREA Press, 2001.

3. I. Foster, C. Kesselman, J. Nick, S. Tuecke. The Physiology of the Grid: An Open Grid Services Architecture for Distributed Systems Integration, Open Grid Service Infrastructure WG, Global Grid Forum, June 22, 2002.

4. I. Foster, C. Kesselman, S. Tuecke. The Anatomy of the Grid: Enabling Scalable Virtual Organizations, International J. Supercomputer Applications, 15(3), 2001.

5. Glue. http://www.themindelectric.com/glue

6. N. Furmento, W. Lee, A. Mayer, S. Newhouse, J. Darlington. ICENI: An Open Grid Service Architecture Implemented with Jini. Proceedings of the IEEE/ACM SC2002 Conference, November 2002.

7. Message Passing Interface Forum. MPI: A Message-Passing Interface Standard. Vers. 1.1, June 1995. MPI-2: Extensions to the Message-Passing Interface, 1997.

8. Java Web Services Developer Pack. http://java.sun.com/webservices

9. S. Tuecke, K. Czajkowski, I. Foster, J. Frey, S. Graham, C. Kesselman. Grid Service Specification: Open Grid Service Infrastructure WG, GGF, Draft 2, 7/17/2002.

10. T. Sandholm, R. Seed, J. Gawor. OGSI Technology Preview Core - A Grid Service Container Framework, OGSI Technology Preview 5, DRAFT 11/08/2002. http://www.globus.org/ogsa/releases/TechPreview

11. OpenMP Architecture Review Board. OpenMP C and $\mathrm{C}++$ Application Program Interface. Version 2.0, March 2002. http://www.openmp.org.

12. L. A. Shepp and Y. Vardi, Maximum likelihood reconstruction for emission tomography. IEEE Trans. Med. Imaging, vol. 1, no. 2, pp. 113-122, 1982.

13. Web Services Invocation Framework. http://ws.apache.org/wsif 